Article

\title{
Challenges and Best Practices for Deriving Temperature Data from an Uncalibrated UAV Thermal Infrared Camera
}

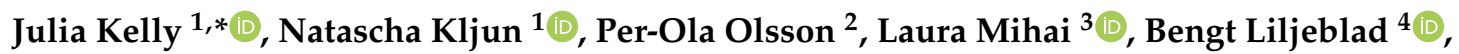 \\ Per Weslien ${ }^{4} \mathbb{D}$, Leif Klemedtsson ${ }^{4}$ and Lars Eklundh ${ }^{2} \mathbb{D}$ \\ 1 Centre for Environmental and Climate Research, Lund University, 22362 Lund, Sweden; \\ natascha.kljun@cec.lu.se \\ 2 Department of Physical Geography and Ecosystem Science, Lund University, 22362 Lund, Sweden; \\ per-ola.olsson@nateko.lu.se (P.-O.O.); lars.eklundh@nateko.lu.se (L.E.) \\ 3 National Institute for Laser, Plasma and Radiation Physics, CETAL, RO77125 Margurele, Romania; \\ laura.mihai@inflpr.ro \\ 4 Department of Earth Sciences, University of Gothenburg, S-405 30 Gothenburg, Sweden; \\ beli@gvc.gu.se (B.L.); per.weslien@gu.se (P.W.); leif.klemedtsson@gu.se (L.K.) \\ * Correspondence: julia.kelly@cec.lu.se; Tel.: +46-46-222-8939
}

Received: 22 January 2019; Accepted: 1 March 2019; Published: 8 March 2019

\begin{abstract}
Miniaturized thermal infrared (TIR) cameras that measure surface temperature are increasingly available for use with unmanned aerial vehicles (UAVs). However, deriving accurate temperature data from these cameras is non-trivialsince they are highly sensitive to changes in their internal temperature and low-cost models are often not radiometrically calibrated. We present the results of laboratory and field experiments that tested the extent of the temperature-dependency of a non-radiometric FLIR Vue Pro 640. We found that a simple empirical line calibration using at least three ground calibration points was sufficient to convert camera digital numbers to temperature values for images captured during UAV flight. Although the camera performed well under stable laboratory conditions (accuracy $\pm 0.5^{\circ} \mathrm{C}$ ), the accuracy declined to $\pm 5^{\circ} \mathrm{C}$ under the changing ambient conditions experienced during UAV flight. The poor performance resulted from the non-linear relationship between camera output and sensor temperature, which was affected by wind and temperature-drift during flight. The camera's automated non-uniformity correction (NUC) could not sufficiently correct for these effects. Prominent vignetting was also visible in images captured under both stable and changing ambient conditions. The inconsistencies in camera output over time and across the sensor will affect camera applications based on relative temperature differences as well as user-generated radiometric calibration. Based on our findings, we present a set of best practices for UAV TIR camera sampling to minimize the impacts of the temperature dependency of these systems.
\end{abstract}

Keywords: UAV; UAS; thermal infrared; FLIR; calibration; temperature; radiometric; remote sensing; vignetting; NUC

\section{Introduction}

Temperature affects environmental processes at all scales, from the global distribution of plants and animals to the photosynthesis of $\mathrm{CO}_{2}$ molecules in leaf chloroplasts. Thermal cameras mounted on unmanned aerial vehicles (UAVs) can provide high resolution and spatially-resolved surface temperature measurements and, therefore, offer a powerful tool for environmental science. UAV-based remote sensing achieves significant improvements in spatial resolution over satellite thermal data ( $<10 \mathrm{~cm}$ compared to $100 \mathrm{~m}$ from the Landsat 8 Thermal Infrared Sensor [1]). The spatial variability 
of temperature across an ecosystem can also be captured over a larger area and at a greater level of detail than when using ground-based thermal imagers or traditional air and soil temperature sensors. As a result, there has been increasing use of UAV thermal imagery for monitoring crop water stress [2-5], mapping evapotranspiration and conductance [6-8], assessing tree health [9], estimating soil moisture [10], estimating stream temperature [11] and for numerous applications outside ecology [12].

Thermal infrared (TIR) cameras measure radiation in the 8-14 $\mu \mathrm{m}$ wavelengths and are known as longwave thermal infrared cameras. The use of TIR cameras on UAVs has been promoted by the availability of miniaturized thermal sensors that are lightweight, low power and fit onto popular UAV platforms. The cost of these cameras varies from $~ € 1000$ to $>€ 10,000$ depending on the resolution of the sensor and the accuracy of the radiometric calibration. The most expensive models offer the highest accuracy temperature measurements but may not be designed specifically for use on UAVs. Consequently, users may opt for cheaper models which are easier to use. Low-cost sensors are generally not radiometrically-calibrated, meaning that they can only provide information on relative temperature differences which are expressed in raw digital numbers (DN, representing the magnitude of the TIR radiance), rather than temperature. Since most TIR camera applications within ecophysiology require absolute temperature measurements, it is valuable to assess whether non-calibrated sensors could be used to provide accurate temperature measurements after a simple user calibration. Moreover, it is important to understand what, if any, factors influence their ability to provide data on relative temperature differences.

Even with radiometrically-calibrated TIR UAV cameras, it is not trivial to derive accurate surface temperature measurements. Manufacturer estimates of temperature accuracy can be up to $\pm 5^{\circ} \mathrm{C}$ (or $5 \%$ of reading). The low accuracy is caused by the sensor (focal plane array, FPA), which is composed of uncooled microbolometers. The sensitivity (gain) and offset of each microbolometer changes with FPA temperature [13]. The microbolometers also receive thermal radiation emitted by the camera interior which can be much greater than that received from the object of interest, resulting in a poor signal to noise ratio [14]. Consequently, radiometric calibration of the camera should account for the thermal radiation produced by different parts of the camera (interior, lens, optics) and correct for the non-uniform response of the individual microbolometers on the FPA. As the specifications of the radiometric calibrations performed by manufacturers are proprietary, it is difficult to ascertain to what extent these factors are accounted for and how [14-16].

During operation, UAV thermal cameras (radiometric and non-radiometric) usually update the offset parameters for all the microbolometers on the FPA after a specified time interval or temperature change by taking an image with the shutter closed [17]. This non-uniformity correction (NUC) ensures a harmonized response signal across the sensor. The NUC is valid for the temperature of the shutter, which is assumed to be the same as that of the rest of the camera interior, lens and optics [13]. However, this assumption is likely not valid for cameras used during UAV flight campaigns as the lens is more exposed to wind than the rest of the camera interior. Furthermore, the camera may experience abrupt changes in ambient temperature during take-off and flight which occur faster than the rate at which the NUC is performed. Smigaj et al. [18] described these effects in a radiometric TIR camera but further examination is warranted for uncalibrated TIR cameras and under UAV flight conditions. Moreover, it is unclear to what extent distortion caused by the lens optics (so-called 'vignetting' [19]) is corrected for by the NUC.

Several other factors also affect temperature measurements performed by TIR cameras including target object emissivity, ambient humidity, temperature of the surrounding objects or sky, and distance between the camera and the target object. All these factors may be accounted for by algorithms included in radiometric cameras and associated software but not in uncalibrated TIR cameras. Aubrecht et al. [20] found that emissivity had the greatest influence on measured temperature. Others have noted that the atmospheric attenuation of thermal radiation can lead to differences in temperature of several $\mathrm{K}$ between the true surface temperature of an object and that measured by a TIR camera $[2,19,21]$. 
Although the temperature dependency effect of uncooled thermal cameras is well-documented in the technical thermography literature [13-15,22], it has only recently received attention in the UAV remote sensing literature [16-18]. These authors have focused on describing methods that minimize the impacts of the temperature dependency effects without a full discussion of the impacts themselves. As a result, UAV TIR camera users may be unaware of the factors that affect surface temperature measurements conducted with TIR cameras and the potentially significant challenges to deriving accurate measurements. Moreover, research has focused almost exclusively on radiometric TIR cameras, with only minor discussions of the temperature dependency effects in uncalibrated cameras (e.g., [3]).

Our study therefore aims to show whether low-cost, uncalibrated UAV TIR cameras can be robust measurement tools for applications in ecophysiology. We focus specifically on testing how the temperature dependency of the camera's sensor affects camera performance in both laboratory and UAV flight conditions. We further assess the stability of surface temperature values derived from a simple empirical line calibration and examine the severity of non-uniformity noise across the sensor and through time. Based on our results we provide a set of best practices for UAV sampling using TIR cameras to help minimize these impacts. These practices will be valuable for those using radiometric TIR cameras since the temperature dependency effects of these systems may not be fully accounted for during radiometric calibration. Users of uncalibrated UAV TIR cameras focusing on relative temperature differences may also find these practices useful for ensuring they produce the most robust results possible.

\section{Materials and Methods}

For this study, all experiments and field campaigns used a non-radiometric TIR camera designed specifically for use with UAVs: the Vue Pro 640 (FLIR Systems, Inc., Wilsonville, USA). The camera has a $13 \mathrm{~mm}$ lens producing a $45^{\circ} \times 37^{\circ}$ field of view and sensor resolution of $640 \times 512$ pixels. It uses an uncooled VOx microbolometer FPA with a spectral range of 7.5-13 $\mu \mathrm{m}$ and an operating temperature range of $-20^{\circ} \mathrm{C}$ to $50^{\circ} \mathrm{C}$. Raw data were captured as 14 bit TIFF images without post-processing. The Vue Pro does not offer the conventional settings for controlling sensitivity, gain and non-linearity correction when using the raw 14 bit TIFF images that are usually available for radiometric cameras. The NUC was enabled during all experiments and occurred at the default rate (when 30\% of pixels display the same DN value or on a 2-3 min interval; personal communication with manufacturer, 18 January 2018).

\subsection{Laboratory Experiments}

We performed four sets of experiments to: (a) assess the time needed for the camera to stabilize after being switched on, (b) understand the relationship between camera DN, surface temperature, and camera temperature, (c) analyze the effect of wind and heating on this relationship, and (d) test for the presence of noise in the data. All experiments were performed in a laboratory. Experiment (b) was additionally repeated in a temperature-controlled incubation chamber. During the experiments, the camera recorded images of a blackbody radiator which filled the camera's field of view (FOV). Blackbody and camera temperature were recorded at $1 \mathrm{~Hz}$ with a PT100 resistance thermometer and a copper-constantan (Type T) thermocouple, respectively. A weather monitor (Kestrel 4500, KestrelMeter, Boothwyn, PA, USA) recorded air temperature, relative humidity and wind speed every $30 \mathrm{~s}$.

\subsubsection{Stabilization Time and Blackbody Curves}

To assess the time needed for camera readings to stabilize after being switched on, images of the blackbody were recorded every $30 \mathrm{~s}$ for $2 \mathrm{~h}$ after activation. The blackbody maintained a constant temperature of $18.4{ }^{\circ} \mathrm{C}\left(\sigma=0.2{ }^{\circ} \mathrm{C}\right)$. Based on the results, we implemented a 1-h stabilization time before all further laboratory experiments. 
To relate camera DN to surface temperature, 20 images of the blackbody were recorded at $6.5^{\circ} \mathrm{C}$ (the lowest temperature reached by the blackbody), then in $5^{\circ} \mathrm{C}$ intervals from $10{ }^{\circ} \mathrm{C}$ to $40^{\circ} \mathrm{C}$. During the experiment, room temperature was kept stable at $20.6^{\circ} \mathrm{C}\left(\sigma=0.3^{\circ} \mathrm{C}\right)$. The experiment was repeated inside a cool incubation chamber (air temperature $=10.8^{\circ} \mathrm{C}, \sigma=0.3^{\circ} \mathrm{C}$, Conviron CMP4030, Conviron, Winnipeg, $\mathrm{MB}, \mathrm{Canada}$ ) to test whether air temperature affected the relationship between camera $\mathrm{DN}$ and surface temperature. The temperature of the blackbody was increased in steps of $5^{\circ} \mathrm{C}$ from $10.3^{\circ} \mathrm{C}$ to $25^{\circ} \mathrm{C}$.

\subsubsection{Wind and Radiative Effects}

A fan and a $500 \mathrm{~W}$ halogen lamp were used to simulate the effects of the wind and solar radiation, respectively, that the camera would experience when mounted on a UAV. The experiment started with the fan blowing towards the camera at $2 \mathrm{~ms}^{-1}\left(\sigma=0.2^{\circ} \mathrm{C}\right)$ for $30 \mathrm{~min}$, while the camera recorded images of the blackbody every $30 \mathrm{~s}$. The experiment was repeated with wind blowing at $3.3 \mathrm{~ms}^{-1}\left(\sigma=0.2^{\circ} \mathrm{C}\right)$. Finally the experiment was repeated with the lamp heating the camera until it reached $45^{\circ} \mathrm{C}$. The lamp was then turned off after $4 \mathrm{~min}$ to avoid overheating the camera and the camera continued recording images of the blackbody for $30 \mathrm{~min}$. During the experiment the blackbody maintained a constant temperature of $19.5^{\circ} \mathrm{C}\left(\sigma=0.3^{\circ} \mathrm{C}\right)$.

\subsubsection{Sensor Noise}

Following Aubrecht et al. [20], we assessed the severity of non-uniformity noise patterns across the sensor by recording images of the blackbody (which maintained a stable temperature of $19^{\circ} \mathrm{C}$, $\left.\sigma=0.1{ }^{\circ} \mathrm{C}\right)$ at $1 \mathrm{~Hz}$ for $30 \mathrm{~min}(\mathrm{n}=1800)$. To test for the presence of vignetting, the deviation of the pixel mean (mean DN of a pixel across all images) from the image mean (mean of all pixels in all images) was plotted according to the location of each pixel on the sensor. To ensure that the observed vignetting effect was not due to the uneven surface heating of the blackbody, we also tested for the vignetting effect in images taken during a UAV flight (see Section 2.2.3). Finally, we tested for the presence of cyclical and temporal patterns of noise which could be related to the automated NUC performed by the camera. For this test, the mean for all pixels across all images was subtracted from each image. Then the mean deviation of each pixel from this total mean was calculated and Fourier transformed to reveal the dominant noise frequencies and their magnitudes.

\subsection{Field Calibration and Validation}

To test the performance of the Vue Pro during UAV flight we conducted four UAV flight campaigns with the camera mounted on an Explorian 8 quadcopter (Pitchup AB, Mölndal, Sweden; Table 1). Campaigns were conducted over the Mycklemossen mire at Skogaryd Research Station $\left(58^{\circ} 23^{\prime} \mathrm{N}\right.$, $12^{\circ} 09^{\prime} \mathrm{E}$, WGS 84) in central Sweden, which is part of the SITES (Swedish Infrastructure for Ecosystem Science, www.fieldsites.se) spectral network. The image capture rate varied between $0.25-1 \mathrm{~Hz}$ among flights while the vertical and horizontal overlap were $\geq 73 \%$ for all flights. The camera's NUC was enabled and occurred at the default rate. The flight conditions varied between partly cloudy and full sun, with air temperatures between $20-28{ }^{\circ} \mathrm{C}$ and wind speeds up to $4 \mathrm{~ms}^{-1}$. Six temperature calibration plates (TCPs, detailed below) were installed at the site for radiometric calibration of the camera by empirical line calibration. We performed a sensitivity test of the calibration method to the number of TCPs used. Finally, the vegetation temperatures derived from the Vue Pro data were validated against measurements from a radiometric thermal camera (A65sc, FLIR Systems Inc., with $13 \mathrm{~mm}$ lens, $\pm 5^{\circ} \mathrm{C}$ accuracy, 'ground camera') installed with a nadir viewing angle $9 \mathrm{~m}$ above the ground on a mast within the UAV flight area. The 'ground camera', produced a FOV covering $45.4 \mathrm{~m}^{2}$, which included a black TCP. The 'ground camera' recorded images every $5 \mathrm{~min}$ and we calculated the mean temperature from 3 images captured during a $15 \mathrm{~min}$ time window centered on the middle of the UAV flight times. 
Table 1. UAV flights conducted with the Vue Pro, including stabilization time of the camera before flight, number of temperature calibration plates (TCPs) used for calibration, number of images used for orthomosaic creation, flight altitude above ground level, and type of data analysis performed.

\begin{tabular}{cccccccccc}
\hline Name & Date & $\begin{array}{c}\text { Start } \\
\text { Time }\end{array}$ & $\begin{array}{c}\text { Stabilization } \\
\text { Time (min) }\end{array}$ & $\begin{array}{c}\text { Flight } \\
\text { Time } \\
\text { (min) }\end{array}$ & $\begin{array}{c}\text { Number } \\
\text { of } \\
\text { Images }\end{array}$ & $\begin{array}{c}\text { Number } \\
\text { of } \\
\text { TCPs }\end{array}$ & $\begin{array}{c}\text { Flight } \\
\text { Altitude } \\
\text { (m) }\end{array}$ & $\begin{array}{c}\text { Resolution } \\
\text { (cm/pixel) }\end{array}$ & $\begin{array}{c}\text { Analysis } \\
\text { A }\end{array}$ \\
\hline A & 28 June 2017 & $10: 44$ & 15 & 11 & 448 & 4 & 50 & 6.3 & Sensitivity \\
B & 11 July 2018 & $19: 52$ & 20 & 8 & 108 & 4 & 61 & 7.5 & Sensitivity \\
C & 12 July 2018 & $14: 47$ & $0^{1}$ & 4 & 39 & 3 & 53 & 6.8 & Validation \\
D & 12 July 2018 & $20: 27$ & 15 & 2 & 58 & 3 & 53 & 6.8 & Validation \\
\hline
\end{tabular}

1 No stabilization time because camera was exposed to direct sunlight for $1 \mathrm{~h}$ prior to flight and heated up accordingly.

\subsubsection{Radiometric Calibration}

To enable radiometric calibration of the camera in the field, six TCPs were installed spread out across the study area (Figure 1a-c). However, because of constraints on flight time due to limited battery power and issues with powering the camera during flight, the test flights only covered 3 or 4 TCPs (Table 1). The TCPs were composed of wooden frames supporting a layer of expanded polystyrene insulation foam and finally a $1 \mathrm{~m} \times 1 \mathrm{~m} \times 0.001 \mathrm{~m}$ sheet of aluminium. The insulation foam reduced short term temperature fluctuations in the TCPs. Large TCPs were necessary to ensure they were easily detectable in the UAV imagery and that a spot size of at least 10 pixels could be extracted from the centre of each TCP [23]. To produce a large temperature range the plates were painted black, grey and white (two of each) with matte paint for concrete. Emissivity measurements on samples of the painted aluminium using a double monochromator and a gold integrating sphere [24] confirmed that the three colors produced high emissivities (black $=0.98$, grey $=0.99$, white $=0.99$ ) in the spectral range of the camera, similar to the values for peatland vegetation ( $>0.94,[25-27])$. Based on the results of Aubrecht et al. [20], the difference in emissivity between the TCPs and vegetation may have caused us to underestimate the vegetation temperature by $\leq 1.25^{\circ} \mathrm{C}$. TCP temperatures were recorded every minute using a thermistor (Model 107, $\pm 0.4{ }^{\circ} \mathrm{C}$ accuracy, Campbell Scientific Inc., Logan, UT, USA) attached to the underside of each aluminium sheet and connected to a CR1000 logger (Campbell Scientific Inc.). We performed an empirical line calibration by regressing the measured TCP temperature against the camera DN recorded for each TCP. A separate calibration was performed for each flight.

\subsubsection{Orthomosaic Creation}

To enable accurate georectification of the orthomosaics produced from the flight campaign data and the geometric calibration of the camera, we installed four ground control points (GCPs) at the site (Figure 1d). The GCPs consisted of a thick piece of aluminum $(60 \times 60 \mathrm{~cm})$ which ensured they were large enough and produced temperatures different enough from the surrounding vegetation to be distinguishable in the TIR images. Black and white tiles arranged in a checkerboard pattern were placed in one corner for use with optical cameras. The GCPs were placed in the four corners of the main study area and their position measured with an RTK-GPS (GRS-1, $1 \mathrm{~cm}$ accuracy, Topcon Corporation, Tokyo, Japan). One corner of each TCP was also measured with the RTK unit so they could be used as GCPs, resulting in a total of 10 GCPs spread across the site.

Orthomosaics from the flights were produced using PhotoScan Professional (Version 1.4.1, Agisoft LLC, St. Petersburg, Russia; now known as Metashape). Before orthorectifying the images, we plotted the mean image DN over time for each flight and excluded all images at the start of the flight, when large changes in DN occurred after takeoff: $17 \%, 0 \%, 6 \%$ and $14 \%$ of the images for Flights A-D, respectively. We also manually excluded blurry images and images with bands of dead pixels: $14 \%$, $19 \%, 46 \%$ and $39 \%$ of the images for Flights A-D, respectively. The total number of remaining images is listed in Table 1. As suggested by Maes et al. [28], internal camera coefficients were optimized during processing in PhotoScan rather than by performing a geometric pre-calibration. There were 
no vignetting correction coefficients for the camera available in the image Exif data. In PhotoScan, the blending method was set to 'mosaic' (each frequency component in the image treated separately) so as to minimize the appearance of seamlines. The reprojection RMSE was $<1$ pixel for all four orthomosaics produced. Orthomosaic DN values were converted to temperature using an empirical line calibration (see Section 2.1.1).
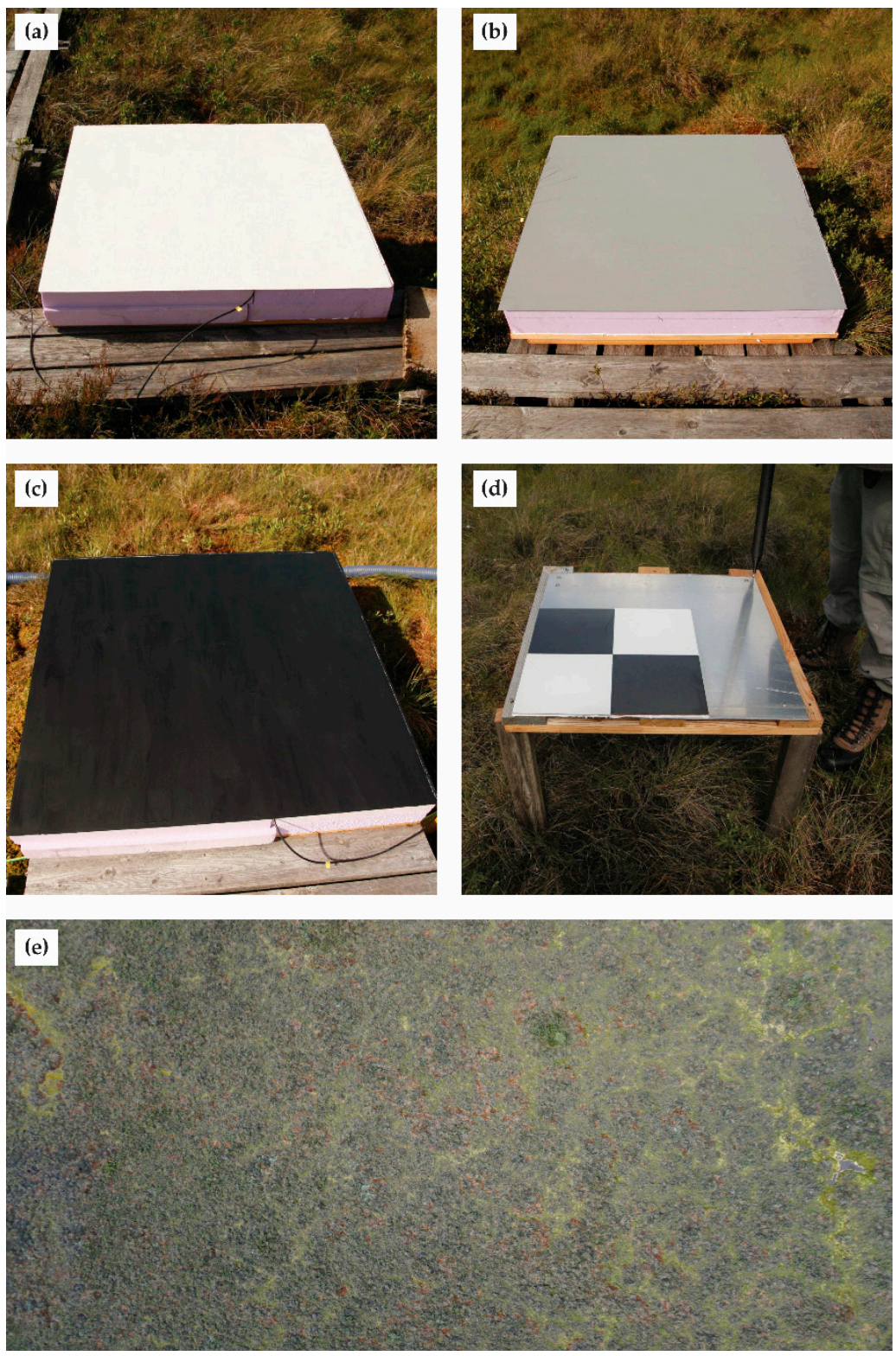

Figure 1. (a-c) Photos of the temperature calibration plates (TCP) used for radiometric calibration, (d) photo of a ground control point (GCP) used for georectification, (e) photo of mire vegetation taken during a UAV flight in June 2017. Using tiles on top of the aluminum GCP created a distinctive ' $\mathrm{L}$ ' shape that facilitated identification of the corner measured with the RTK-GPS, as shown in (d), and enabled the GCPs to be used for both optical and TIR UAV cameras.

\subsubsection{Validation}

We used the two flights that covered the highest number of TCPs (4, Flights ' $\mathrm{A}$ ' and 'B') to assess the robustness of the empirical line calibration method. To test the sensitivity of the method to the number of TCPs used, we calculated the difference in mean scene temperature (all pixels in an orthomosaic) when the calibration was performed using only 3 or 2 TCPs instead of all 4 TCPs. 
To validate our temperature estimates from the UAV TIR camera we used the two flights on 12 July 2018 (Flights ' $C$ ' and ' $D$ ') when 'ground camera' data were available. To compare UAV TIR camera temperature estimates with those measured by the 'ground camera', we defined six regions of interest (ROIs, $45 \mathrm{~cm} \times 45 \mathrm{~cm}$ ) within the FOV of the 'ground camera' that contained homogeneous vegetation. The position of the ROIs was surveyed using an RTK-GPS to enable their identification in the orthomosaics. A linear regression of the mean temperatures of all the pixels within each ROI of the ground and the UAV TIR camera $(n=12)$ showed a significant positive relationship $\left(R^{2}=0.96\right.$, $\alpha<0.001)$.

We also validated our laboratory analysis of the vignetting effect (see Section 2.1.3.) to ensure that any vignetting detected was not due to the uneven surface heating of the blackbody. Using Flight A (the longest flight and closest to solar noon), we selected all images that showed the homogeneous mire surface. We then calculated the column- and row-wise means across all images and plotted them against the respective row or column number to examine whether the vignetting effect was present. Using column- and row-wise means minimized noise and patterns related to the vegetation present in the imagery.

\section{Results}

\subsection{Laboratory Experiments}

\subsubsection{Stabilization Time}

The amount of time needed for the camera to warm-up and produce stable DN values was assessed from repeated images of the blackbody taken over two hours after activation (Figure 2). The Vue Pro showed marked changes in DN values over the course of the experiment, with an abrupt increase of $>1000 \mathrm{DN} 10 \mathrm{~min}$ after activation (Figure 2), equivalent to an increase in temperature of $>60{ }^{\circ} \mathrm{C}$ (Equation (2)). We repeated the experiment and saw a similar sudden jump in DN values after 9 min (Figure A1). In both cases, the jump in DN values occurred at a camera temperature of $29^{\circ} \mathrm{C}$. We also observed a similar pattern during the stabilization time of Flight 'A' (Figure A2).

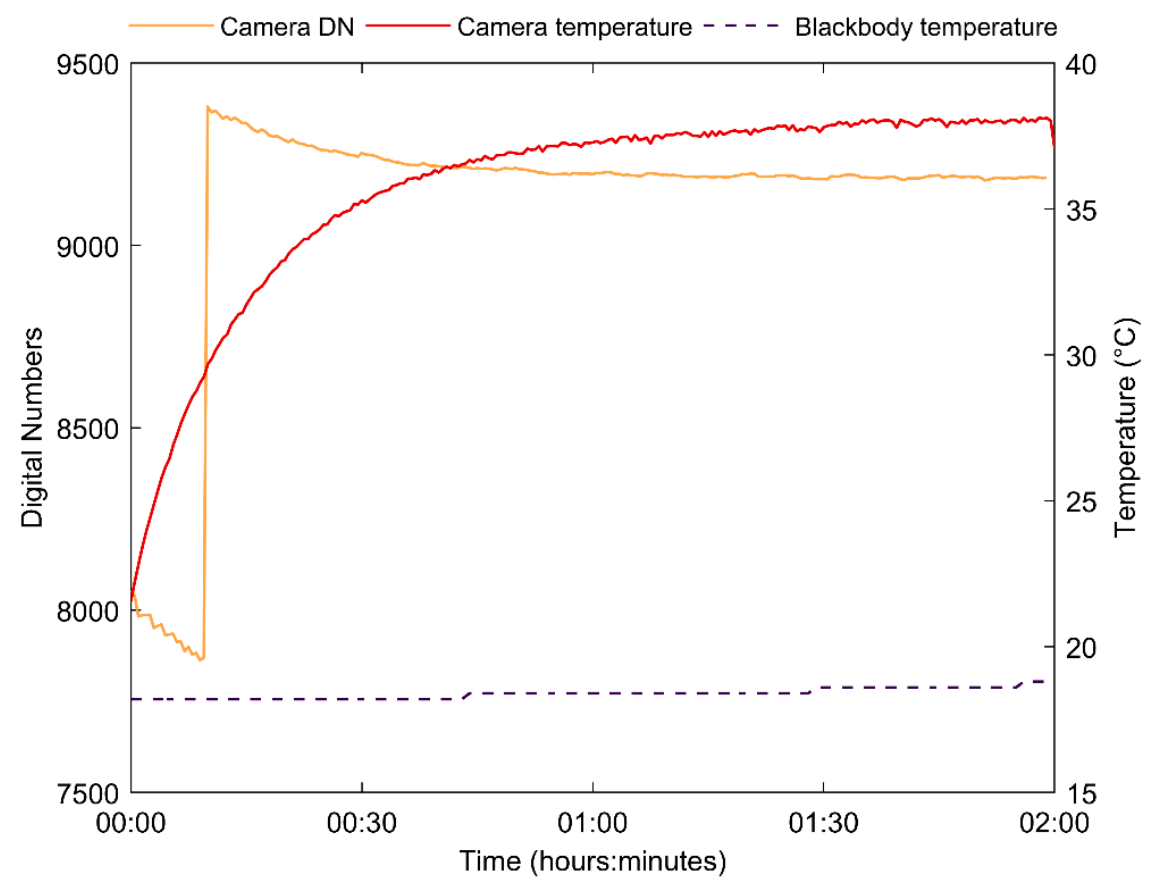

Figure 2. Time series of camera digital numbers (DN), temperature of the camera and blackbody temperature for the first two hours after camera activation. 


\subsubsection{Blackbody Curves}

The relationship between the camera $\mathrm{DN}$ and blackbody temperature was assessed at two different ambient air temperatures $\left(10{ }^{\circ} \mathrm{C}\right.$ and $21^{\circ} \mathrm{C}$; Figure 3), which led to a large difference in camera temperature $\left(20.3^{\circ} \mathrm{C}, \sigma=0.1\right.$ and $\left.39^{\circ} \mathrm{C}, \sigma=0.3\right)$. A simple linear fit represented the blackbody curves best $\left(R^{2}=0.99\right.$ in both cases),

$$
\begin{aligned}
& D N=25 T_{b b}+7593, \\
& D N=23 T_{b b}+8748,
\end{aligned}
$$

where Equations (1) and (2) were for ambient air temperatures $10^{\circ} \mathrm{C}$ and $21^{\circ} \mathrm{C}$, respectively, and $\mathrm{T}_{\mathrm{bb}}$ was the temperature of the blackbody. For all object temperatures, variation in mean $\mathrm{DN}$ among images captured at the same temperature step was low ( $\sigma<3.4 \mathrm{DN}$, Figure 3 ) and equivalent to $\sim 0.16{ }^{\circ} \mathrm{C}$ (Equations (1) and (2)). The camera was thus capable of detecting changes in object temperature of at least $\geq 1{ }^{\circ} \mathrm{C}$ when a stable camera temperature was maintained. However, the results clearly showed that camera temperature had a strong impact on measured DN values, with a higher camera temperature resulting in a higher $\mathrm{DN}$ value recorded for a given blackbody temperature.

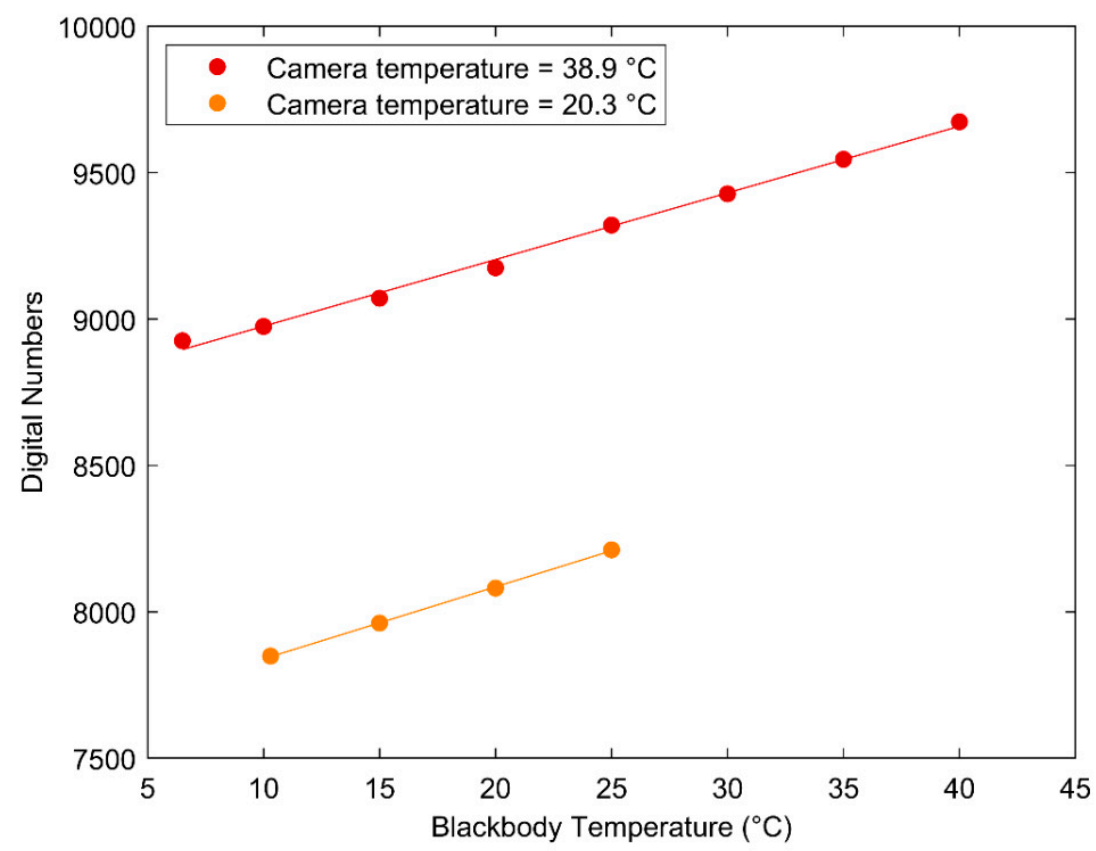

Figure 3. Blackbody curves for the Vue Pro conducted at a room temperature of $21{ }^{\circ} \mathrm{C}$ (camera temperature $=38.9{ }^{\circ} \mathrm{C}, \sigma=0.3^{\circ} \mathrm{C}$ ) and in an incubation chamber at $10{ }^{\circ} \mathrm{C}$ (camera temperature $=20.3^{\circ} \mathrm{C}, \sigma=0.1{ }^{\circ} \mathrm{C}$ ). Error bars showing $\pm \sigma \mathrm{DN}$ for the 20 images recorded at each blackbody temperature step are not visible as $\sigma<3.4 \mathrm{DN}$.

\subsubsection{Wind and Radiative Effects}

Both wind treatments and the heat treatment had a pronounced effect on camera DN values and in no case did DN values recover to their original values by the end of the $30 \mathrm{~min}$ treatment period (Figure 4). During the two wind treatments (Figure 4a,b), the camera response changed by more than $500 \mathrm{DN}$, which is equivalent to a temperature change of $>20^{\circ} \mathrm{C}$ (Equations (1) and (2)). The change in $\mathrm{DN}$ values was less pronounced during the heat treatment (Figure 4c), and occurred in the opposite direction to that seen during the wind treatments. To minimize the risk of damaging the camera, the heat treatment was only applied during the first four minutes of the treatment period, at which point the camera temperature was approaching the upper limit of its operating range $\left(50^{\circ} \mathrm{C}\right)$. 
During the three experiments, the camera DN was negatively related to camera temperature, which contrasted with the blackbody curves (Figure 3). We cannot explain why the relationship is reversed, other than to speculate that the internal system of the camera was overcompensating for the sudden change in sensor temperature during the wind and heating experiments. The internal operation of these cameras is considered sensitive proprietary information (personal communication with manufacturer, 18 January 2018). Furthermore, the DN values did not stabilize until 15 min after the start of the treatment. The DN values from UAV flights with a duration $<15$ min could therefore be severely affected by wind.

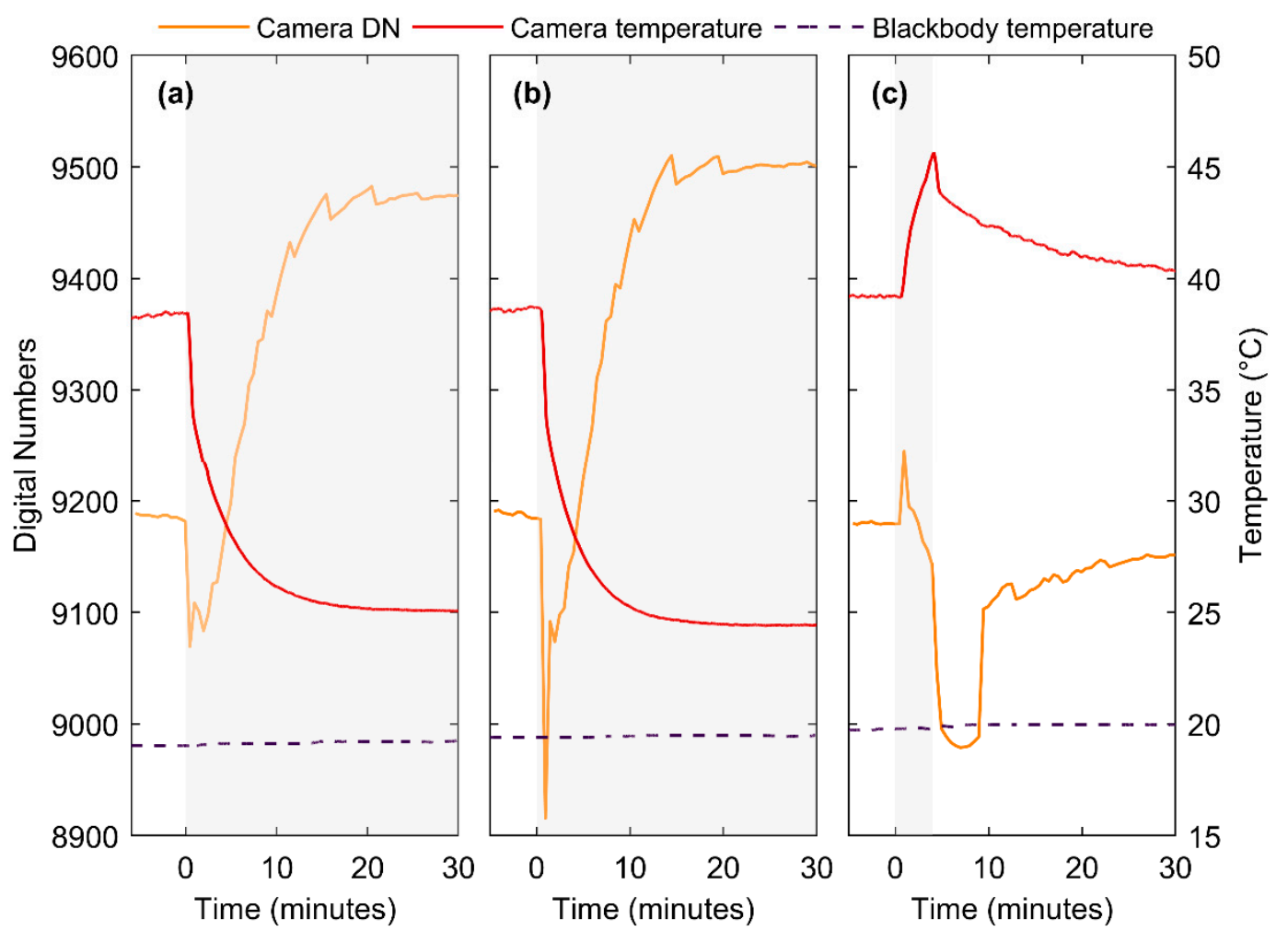

Figure 4. Simulation of the effect of wind and radiative heating on the camera while imaging a blackbody at a constant temperature, (a) with wind speed of $2 \mathrm{~ms}^{-1}$, (b) with wind speed of $3.3 \mathrm{~ms}^{-1}$ and (c) with radiative heating from a $500 \mathrm{~W}$ lamp. Data are plotted using a $30 \mathrm{~s}$ moving average for clarity. The shaded area shows the treatment duration.

\subsubsection{Sensor Noise}

The highest magnitude of noise frequencies in the camera signal occurred at two cycles/hour and at six cycles/hour (Figure 5a). The former was likely a consequence of the experiment duration (30 $\mathrm{min}$ ), while the latter could have resulted from the automatic recalibration of the camera. In any case, the magnitudes of all frequencies were small and thus should only have had a weak impact on the camera signal quality as long as the camera temperature remained stable.

Weak non-uniformity noise was present across the sensor (Figure 5b). It was visible as a pattern of vertical and horizontal stripes where neighboring pixels exhibited similar correlation to the central pixel, i.e., they had similar patterns of deviation from the image mean DN. Correlation values remained mostly between -0.5 to 0.5 indicating a low impact on image quality. However, prominent vignetting (in which DN values at the edge of the sensor are lower than those at the center) was visible on images taken in the laboratory (Figure 6a). The DN values ranged between 9124 and 9182 which is equivalent to a temperature range of $2.6^{\circ} \mathrm{C}$ across the sensor (Equation (2)). 

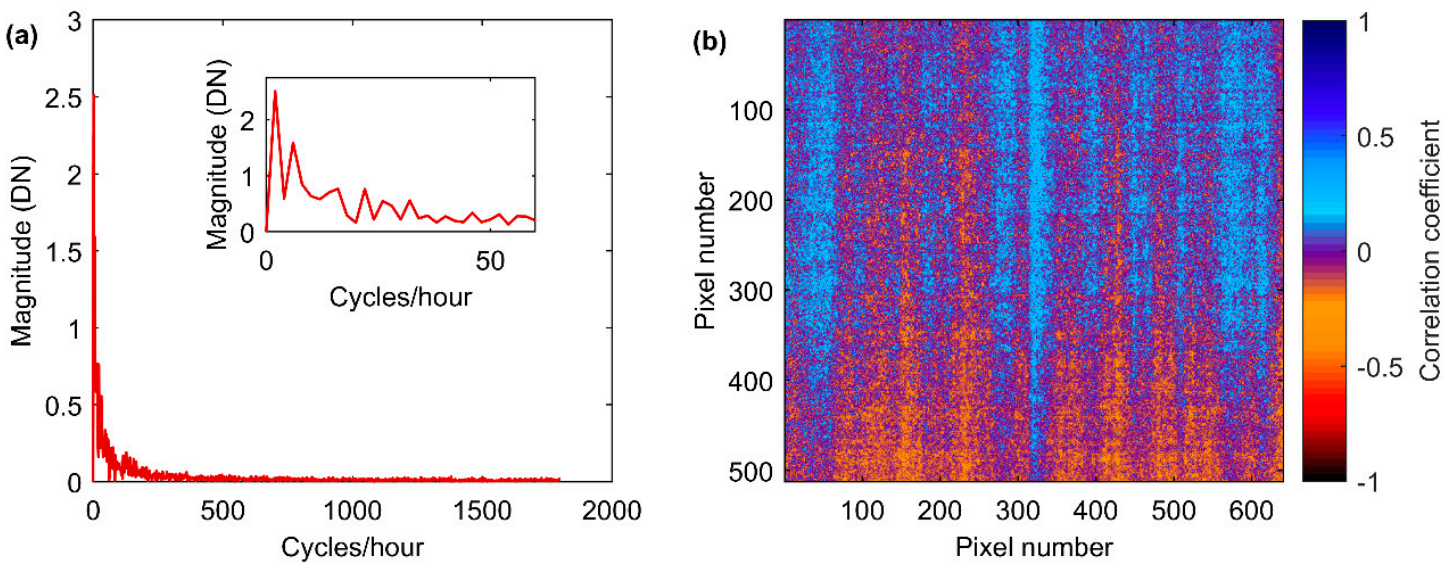

Figure 5. (a) Frequency magnitudes of sensor noise over $30 \mathrm{~min}(1 \mathrm{image} / \mathrm{s}, \mathrm{n}=1800)$ using Fourier transform (see Section 2.1.3). The inset shows the highest magnitude frequencies. (b) Pearson's correlation coefficients between every pixel and the central pixel on the sensor. Neighboring pixels with similar coefficients exhibited similar patterns of deviation of DN from the image mean DN (i.e., non-uniformity noise).

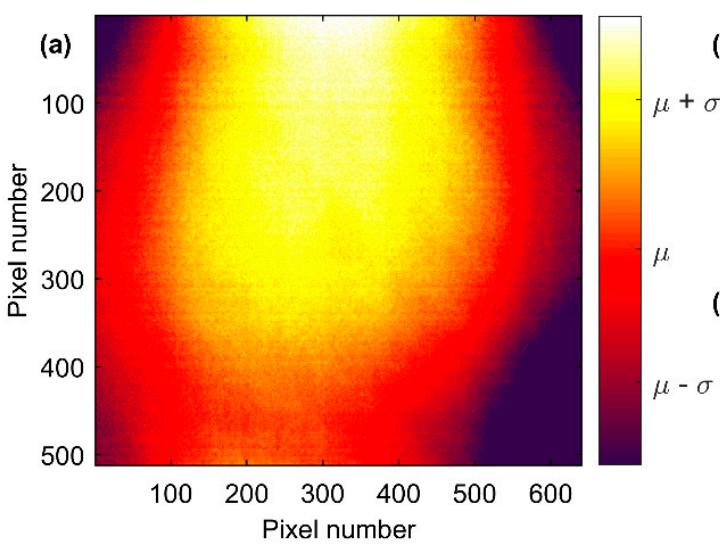

(b)

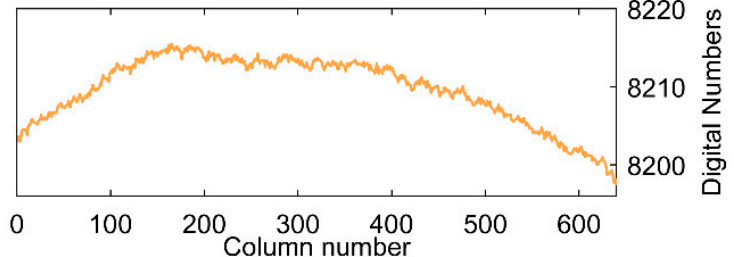

(c)

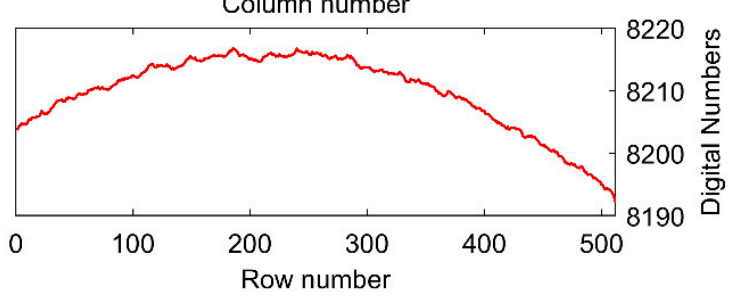

Figure 6. Analysis of the vignetting effect in images of (a) a stable blackbody, with mean image $\mathrm{DN}=9167(\sigma=8.26 \mathrm{DN})$; and $(\mathbf{b}-\mathbf{c})$ images taken of a mire surface during Flight A. (b) shows column mean DN while (c) shows row mean DN (see Section 2.2.3. for details). Row number 0 corresponds to the top of the sensor and column number 0 to the left side of the sensor.

\subsection{Field Calibration and Validation}

To test the performance of the Vue Pro in field conditions, we conducted four UAV flights over a mire ecosystem. The resulting orthomosaics of $\mathrm{DN}$ values were converted to temperature using an empirical line calibration based on measured temperature values from the TCPs at the site (Figure 7a-d). Similar to the laboratory experiments (Figure 3), there was a significant linear relationship between TCP temperature and DN values for all flights (Table 2). Although the slope of the regressions was similar between the flights, the y-intercept differed substantially, demonstrating the need for a separate calibration for each flight.

Despite allowing the camera to stabilize for at least $10 \mathrm{~min}$ prior to most flights, three out of the four flights had strong, positive trends in image mean DN during the flight (Figure 7e-h). Based on the calibration equations (Table 2), these trends are equivalent to changes in mean image temperature of between $\sim 0.5^{\circ} \mathrm{C} / \mathrm{min}$ to $\sim 2{ }^{\circ} \mathrm{C} / \mathrm{min}$ of flight time. The flight with the shortest stabilization time had the strongest trend (Flight $\mathrm{C}$, cf. Tables 1 and 2).

As the mire vegetation is relatively homogeneous (cf. Figure 1e) and the flight durations were $<10 \mathrm{~min}$, we argue that the changes in mean image DN were largely due to camera performance 
rather than vegetation temperature changes between images. The one, notable exception was Flight $B$ (Figure 7f), where both maxima and minima in image mean DN values coincided with UAV turns at the end of each flight line. These large shifts in image mean DN were likely due to the change in camera angle as the UAV turned which caused it to capture a larger portion of the cool shadows or warm tree trunks from the forest that borders the mire. However, this did not explain the consistent decrease or increase in image mean DN during each flight line. The pattern of image mean DN from flight B contrasted to that experienced during Flight D (Figure 7h), where only sharp decreases in image mean DN occurred soon after each UAV turn. This pattern could have been related to the change in temperature (increase in wind) that the camera experienced during a turn or could have resulted from the automated NUC.

We also assessed whether the vignetting effect was present on images taken during Flight A. Figure $6 \mathrm{~b}, \mathrm{c}$ show declines in average $\mathrm{DN}$ value towards the edge of the sensor, which are equivalent to a change in temperature of $1.2^{\circ} \mathrm{C}$ (Table 2). This pattern was similar to that seen during the laboratory analysis (Figure 6a), thus confirming the existence of a significant vignetting effect.
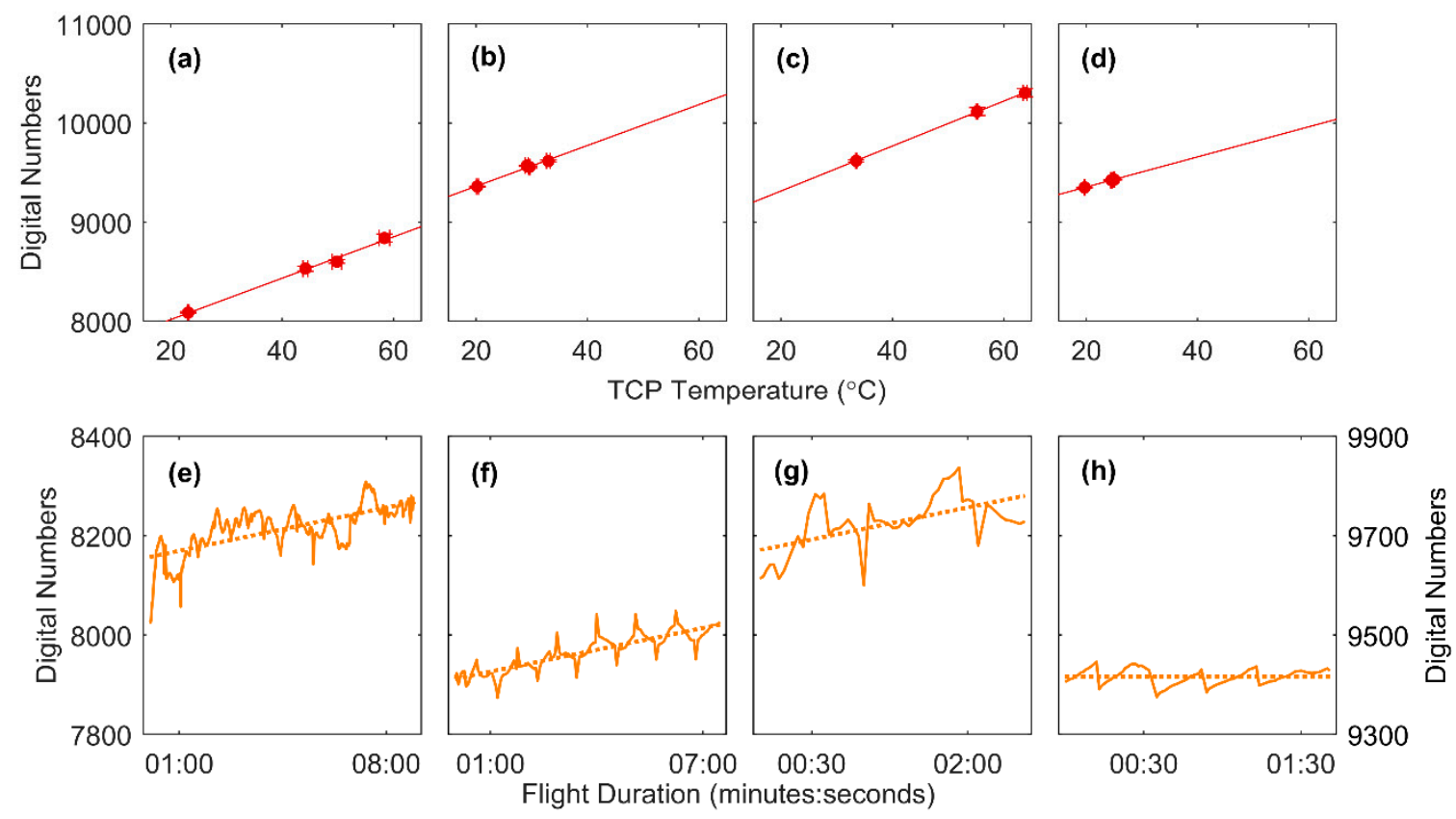

Figure 7. Empirical line calibrations using measured TCP temperatures (red circles) for Flights A-D (a-d, respectively), error bars denote $\pm \sigma$. Horizontal error bars are $\leq 0.9^{\circ} \mathrm{C}$ and vertical error bars are $<42 \mathrm{DN}$. Mean digital number values over the duration of the flight for Flights A-D (e-h, respectively), including linear regression line (dashed orange line). Note that (e) uses the left y-axis but that (f-h) use the right $y$-axis and flight duration varies for plots (e) to (h).

Table 2. Linear regression for calibration of TCP temperature against DN ('Calibration') and linear regression of mean image $\mathrm{DN}$ against flight duration (in minutes, 'Mean image $\mathrm{DN}^{\prime}$ ), for the four UAV flights. Number of TCPs used for the calibration regressions are listed in Table 1. Sample size (i.e., number of images) used for the 'Mean $\mathrm{DN}^{\prime}$ ' regressions are $\mathrm{n}=538$ (Flight $\left.\mathrm{A}\right), \mathrm{n}=134(\mathrm{~B}), \mathrm{n}=53(\mathrm{C})$ and $n=80(D)$.

\begin{tabular}{ccccccc}
\hline Flight & Calibration & $\mathbf{R}^{\mathbf{2}}$-adj & $\boldsymbol{p}$-Value & Mean image $\mathrm{DN}$ & $\mathbf{R}^{\mathbf{2}}$-adj & $\boldsymbol{p}$-Value \\
\hline $\mathrm{A}$ & $\mathrm{DN}=20.8(\mathrm{TCP})+7601$ & 0.99 & $<0.01$ & $\mathrm{DN}=12.4(\mathrm{mins})+8157$ & 0.46 & $<0.001$ \\
$\mathrm{~B}$ & $\mathrm{DN}=20.6(\mathrm{TCP})+8950$ & 0.97 & $<0.01$ & $\mathrm{DN}=14.6($ mins $)+9411$ & 0.74 & $<0.001$ \\
$\mathrm{C}$ & $\mathrm{DN}=22.7(\mathrm{TCP})+8861$ & 0.99 & $<0.01$ & $\mathrm{DN}=43.0($ mins $)+9670$ & 0.34 & $<0.001$ \\
$\mathrm{D}$ & $\mathrm{DN}=15.2(\mathrm{TCP})+9050$ & 0.99 & $<0.05$ & $\mathrm{DN}=0.37($ mins $)+9416$ & 0 & $>0.05$ \\
\hline
\end{tabular}


The sensitivity analysis showed increasing deviation from the original empirical line calibration when using fewer TCPs and TCPs with a smaller temperature range for the calibration (Figure 8). To quantify the impact of the number of TCPs on the calibration, we calculated the difference between mean orthomosaic temperature derived using four TCPs, compared to using only three or two TCPs (Table 3). The calibration was accurate to within $3{ }^{\circ} \mathrm{C}$ when using only three TCPs instead of four (Table 3). Much larger differences in mean scene temperature arose when using only two TCPs for the calibration. This was due to the small temperature range covered by the remaining two TCPs. For example, for Flight A, the two TCPs that caused the largest deviation from mean orthomosaic temperature only covered a $5.6{ }^{\circ} \mathrm{C}$ temperature range, whereas the two TCPs causing the lowest deviation covered a $14.1^{\circ} \mathrm{C}$ range. The largest deviation from the mean orthomosaic temperature when only two TCPs were used occurred for Flight A. This is likely because the empirical line calibration was less accurate for Flight $A$ due to the greater difference between the average temperatures of the vegetation and the TCPs for Flight A compared to Flight $\mathrm{B}\left(\sim 15^{\circ} \mathrm{C}\right.$ and $\sim 10^{\circ} \mathrm{C}$, respectively).
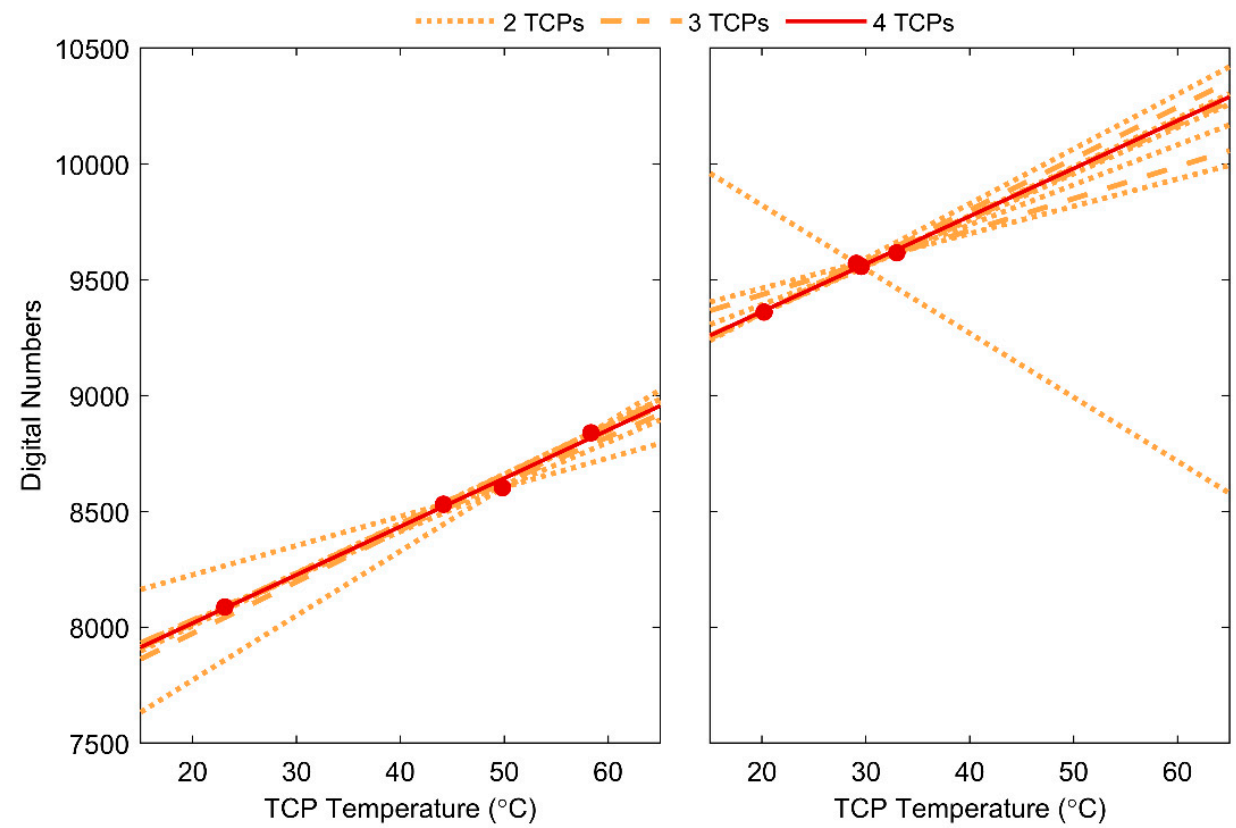

Figure 8. Sensitivity analysis of the TCP calibration for Flights A (a) and B (b). Each flight included four TCPs (red circles, error bars not shown as $\sigma<41 \mathrm{DN}$ and $<0.97^{\circ} \mathrm{C}$, red regression line). The sensitivity of the regression between TCP temperature and $\mathrm{DN}$ was tested by plotting regression lines using only two (dotted line) or three (dashed line) TCPs instead of the original four.

Table 3. Deviation of mean orthomosaic temperature from the original ('4 TCP mean') when calculated using three or two TCPs, respectively. Max 3(2) $\Delta=$ maximum difference in mean temperature between '4 TCP mean' and mean calculated using 3(2) TCPs. Min 3(2) $\Delta=$ minimum difference in mean temperature between ' 4 TCP mean' and the mean calculated using 3(2) TCPs. All numbers are absolute values.

\begin{tabular}{cccccc}
\hline Flight & $\mathbf{4} \mathbf{T C P}$ Mean $\left({ }^{\circ} \mathrm{C}\right)$ & $\operatorname{Max} \boldsymbol{\Delta} \mathbf{3}\left({ }^{\circ} \mathrm{C}\right)$ & $\operatorname{Min} \boldsymbol{\Delta} \mathbf{3}\left({ }^{\circ} \mathrm{C}\right)$ & $\operatorname{Max} \boldsymbol{\Delta} \mathbf{2}\left({ }^{\circ} \mathrm{C}\right)$ & $\operatorname{Min} \boldsymbol{\Delta} \mathbf{2}\left({ }^{\circ} \mathrm{C}\right)$ \\
\hline A & 29.5 & 1.4 & 0.15 & 10.3 & 0.17 \\
B & 25.1 & 2.8 & 0.02 & 7.7 & 0.17 \\
\hline
\end{tabular}

The UAV camera generally overestimated the temperature of the vegetation compared to the measurements conducted by the 'ground camera', with a maximum temperature difference of $5.1^{\circ} \mathrm{C}$ (Figure 9). The apparent overestimation of vegetation temperature by the UAV camera could be due to uncertainty in the radiometric calibration of the 'ground camera', which has a large error range $\left( \pm 5^{\circ} \mathrm{C}\right)$. Indeed, the 'ground camera' underpredicted the temperature of the TCP within its FOV by $4.0^{\circ} \mathrm{C}$ and 
$4.1{ }^{\circ} \mathrm{C}$, during the noon and evening flights, respectively, based on temperature readings from the TCP thermistor. Since the UAV camera temperature estimates are directly tied to the TCP temperature measurements, the difference between the 'ground camera' and thermistor temperature readings would account for the discrepancies between the ground and UAV camera measurements during both flights. These discrepancies could also result from the orthomosaic creation process in PhotoScan, in particular the resampling of images using bilinear interpolation and the use of the 'mosaic' blending mode which derives DN values from several images (with different viewing angles) and pixels from all parts of the image frame. As a result, edge pixels, where the vignetting effect was strongest, would also have been included. Other factors that could lead to differences in vegetation temperature recorded by the UAV and 'ground camera' include uncertainty in the temperature calibration of the UAV imagery based only on three TCPs (Figure 8 ) and a low signal to noise ratio. Given these uncertainties, we estimate that the temperature of the vegetation measured by the UAV camera was accurate to within $\pm 5^{\circ} \mathrm{C}$.

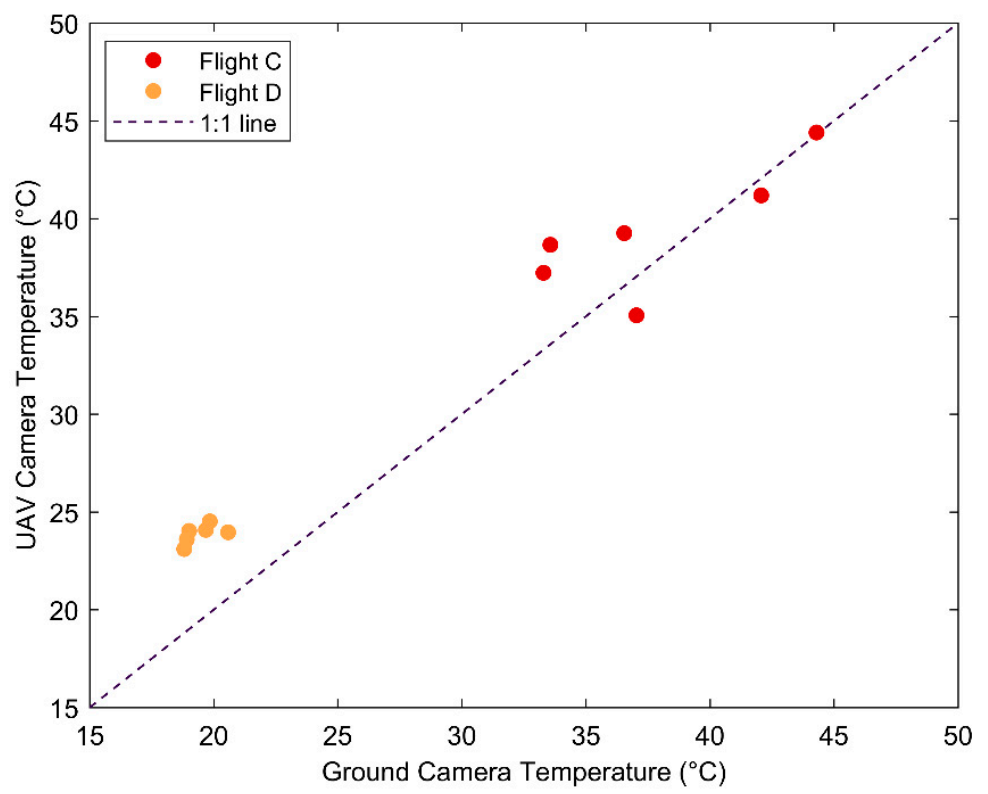

Figure 9. Validation of vegetation temperature recorded by the UAV camera with vegetation temperature recorded by the 'ground camera' for six ROIs during Flights C and D.

\section{Discussion and Recommendations}

\subsection{Stabilization Time}

The dramatic shift in camera DN after activation (see Figure 2) highlights the need to allow at least $15 \mathrm{~min}$ (but preferably one hour) for the camera measurements to stabilize after activation. Though other studies have demonstrated the need for between $30 \mathrm{~min}$ to one hour stabilization time for radiometric UAV TIR cameras, they did not show the same abrupt change in DN values after camera activation [2,9]. The shift we observed may have been a result of the particular type of camera used. In the field, the time allowed for stabilization was limited by the number and longevity of UAV batteries available. Camera users thus face a trade-off between allowing the camera to stabilize and the length of the flight (and thus the size of the area covered) unless an alternative power source or a sufficient number of charged batteries are available. After allowing the camera to stabilize on the ground after activation, extra flight lines could be added at the beginning of the UAV flight to allow the camera to adjust to the air temperature and wind conditions experienced during flight. An extra flight time of $15 \mathrm{~min}$ or more if possible (battery dependent) may counter the effects of temperature drift noted in the wind experiment (discussed below). 


\subsection{Camera Accuracy and Vignetting}

After warming up, the camera provided temperature readings accurate to at least $1{ }^{\circ} \mathrm{C}$ (Figure 3 ) within the temperature range tested and under stable ambient conditions. Although there were no significant non-uniformity or temporal noise patterns (Figure $5 a, b$ ), a strong vignetting effect was evident across the sensor, both under stable conditions (Figure 6a) and during UAV flight (Figure 6b). This resulted in temperature differences of up to $2.6^{\circ} \mathrm{C}$ between pixels at the centre and the edge of the sensor. Vignetting has several causes including: the aperture of the sensor partly blocking the incident radiation, a decline in irradiance depending on the angle and distance between the lens and the image sensor, misalignment of the sensor and lens, as well as the refraction of incident radiation by the lens [29]. The strength of the vignetting effect generally decreases with focal length [29], but UAV cameras often use wide-angle lenses to produce a large FOV. It is especially problematic for applications where absolute or relative temperature differences are compared within a single frame, since objects towards the edge of the frame will appear colder (have lower DN) than those in the center. Vignetting can also introduce artefacts when UAV images are stitched together during orthomosaic creation [30].

A variety of methods have been suggested for correcting vignetting, but image-based techniques using a uniform radiation source to create a look up table of per-pixel correction values or a vignetting polynomial have been most widely applied for UAV cameras [31]. Meier et al. [19] used a similar pixelwise approach for a ground-based TIR camera. Some photogrammetry software packages also include vignetting correction options, e.g., by modelling the camera optics using the coefficients included in image headers [32]. Hoffmann et al. [8] found that when using Photoscan to create orthomosaics for TIR UAV imagery, using the 'mean value' composition setting during orthomosaic generation led to similar results as to when an orthomosaic was produced excluding all image edges (to exclude the vignetting effect). We recommend using only the central portion of the frame for applications where individual images are analyzed, or undertaking a vignetting correction if the necessary equipment is available.

\subsection{Relationship between DN and Surface Temperature}

The blackbody curves (Figure 3) showed that a linear regression was sufficient to model the relationship between camera DN and measured temperature, suggesting that an empirical line calibration could be used to calibrate the camera in the field. Indeed, models of the relationship between incident radiant flux and microbolometer response are often simplified to a linear form [22,33], confirming the appropriateness of an empirical line calibration. The empirical line calibration is a simple, commonly applied method for the calibration and validation of UAV spectral data [31], including the calibration of non-radiometric TIR UAV cameras [3,34,35]. One advantage of this method is that it circumvents the need to correct for the atmospheric attenuation of TIR radiation, which can lead to temperature differences of several degrees Celsius between true surface temperature and that measured by a TIR camera $[2,19,21]$. By using this type of linear calibration we also respect the principle of model parsimony and avoid overfitting a more complex calibration model, particularly since there were only a small number of field calibration points available. Another commonly implemented calibration method for TIR cameras is an exponential model based on Planck's law $[14,20,36]$ while Ribeiro-Gomes et al. [16] proposed using a neural network for calibration.

We found a clear linear relationship between measured TCP temperature and camera DN for the four test flights (Table 2), though the small number of available points reduced the power of the regression significance test [37]. The slope (gain) of the calibration equations was similar between flights, but there were marked differences in y-intercept (offset or dark current) due to changes in camera temperatures during each flight. It is therefore vital to perform a calibration or validation for each flight individually, and thus to have ground calibration/validation points available. A laboratory calibration of the camera based on blackbody curves is only useful if the calibration is performed at multiple camera temperatures (spanning the range expected in the field, e.g., see $[15,16]$ ) and if the 
camera temperature is recorded during flight (or is included in the image metadata, which is not the case for the Vue Pro).

\subsection{Wind Effects and Temperature Drift}

Camera temperature was non-linearly related to DN when the camera was exposed to wind and radiative heating in the laboratory (Figure 4a,b). Although sensor temperature declined exponentially during the wind treatments, DN values first sharply decreased then rapidly increased to well above the pre-treatment values. Thus both wind treatments caused changes in camera DN equivalent to a change in measured temperature of $>20^{\circ} \mathrm{C}$. Smigaj et al. [18] conducted a similar test of the effect of wind on a radiometric UAV TIR camera but did not note significant changes in performance. The contrasting results could have occurred because their experiment did not expose the camera to sustained wind or did not cause a pronounced change in camera temperature. The radiometric calibration of their camera may also have included a correction for the temperature drift experienced.

The impacts of camera temperature on DN values were also evident during the four UAV test flights we performed. Firstly, images from UAV take-off and the start of the flight had to be excluded during orthomosaic creation because of large changes in DN value, probably a consequence of the sudden impact of wind on the camera. Secondly, three of the flights showed significant positive trends in mean image DN value (Figure 7e-g). Mesas-Carrascosa et al. [17] argued that increasing DN values during a UAV flight with a TIR camera were the result of sensor temperature drift, whereby as the camera warmed up it recorded higher surface temperatures values. Their findings implied that any cooling effect of wind was not strong enough to prevent the temperature rise of the sensor during the flight, though no data on sensor temperature were provided. It must be noted that their experiments were performed whilst the NUC was disabled, whereas the NUC was enabled during our flights.

There is a contradiction between the temperature drift effect which assumes a positive relationship between camera temperature and DN, and the results of our wind experiment which showed the opposite. The negative relationship we observed between the camera DN and temperature could have been due to temperature differences between the camera exterior (measured during the experiment) and camera interior (used for NUC correction and the main cause of temperature drift). It could also have stemmed from the non-linear and temperature-dependent gain and offset parameters of the FPA microbolometers. Although the output voltage of a microbolometer can be linearly related to incident radiant flux, the offset and gain parameters used in this equation are temperature-dependent, which has to be corrected using polynomial models in addition to performing a 1-point offset correction using the NUC [14]. We do not know if or how the temperature dependency of the offset and gain parameters are accounted for in our camera model due the proprietary nature of these processes. Budzier et al. [14] suggested that some camera manufacturers use a predictive model based on past changes in temperature, which may explain the 'overcompensation' of the NUC during our wind experiment if the model expected a greater reduction in temperature than that which actually occurred. The temperature changes may also have been too large to be compensated by the NUC or the interval between NUCs may have been too long.

To avoid issues related to trends in DN during UAV flight and the influence of wind, we recommend that UAV TIR camera users enable NUC correction and (where possible) set a short time interval between NUC occurrence. The camera could also be mounted with a custom-made wind shield or within the body of a fixed-wing UAV to minimize the impact of wind during flight. Gómez-Candón et al. [3] reduced issues of thermal drift by flying over ground calibration targets at the start, middle and end of their UAV flights and deriving separate calibration equations for images closest to each overpass as well as only selecting images taken immediately after an NUC had occurred. However, this would limit the total area that could be covered during a flight given the need for repeated overpasses of the ground calibration targets. Mesas-Carrascosa et al. [17] on the other hand suggested a method for correcting for temperature drift during post-processing, based on the features used for structure-from-motion photogrammetry during orthomosaic creation. 
Correction for temperature drift effects may be necessary for analyses based on changes in relative temperature (as well as those using absolute temperature values) because trends in $\mathrm{DN}$ will be apparent in the orthomosaics produced and will cause problems for temporal analyses of relative temperature differences.

\subsection{Field Validation}

Based on the results of the sensitivity analysis, we argue that three is the minimum number of points that should be used for an empirical line calibration. When only two points were used, large deviations in orthomosaic mean temperature occurred $\left(>10^{\circ} \mathrm{C}\right)$. The magnitude of the deviation depended on the temperature range covered by the two points, with the largest deviation occurring, as expected, when the two points with the smallest temperature range were used for the calibration. In addition, the calibration targets should ideally produce temperatures similar to the range of temperatures expected within the scene, to minimize uncertainties when converting DN to temperature outside this range. In our case, when using only two TCPs, the temperatures of the two selected TCPs were in some cases substantially $\left(>10^{\circ} \mathrm{C}\right)$ warmer than the vegetation temperature, resulting in higher uncertainty in the empirical line calibration at the vegetation temperature than when a larger number of TCPs were used with a temperature closer to that of the vegetation.

The validation of the radiometric calibration against measured vegetation surface temperature suggests that the UAV TIR data was accurate to approximately $\pm 5^{\circ} \mathrm{C}$, though uncertainties in the UAV and ground TIR camera measurements prevented us from making a more precise assessment. Using the ground TIR camera had several advantages over other validation methods (e.g., handheld infrared thermometers), such as being able to collect validation data concurrently with the UAV flights and over a larger area (ground camera FOV $=45.4 \mathrm{~m}^{2}$ ). In a similar calibration of a non-radiometric UAV TIR camera, Gómez-Candón et al. [3] also achieved accuracies within a few degrees Celsius (though generally closer to $1^{\circ} \mathrm{C}$ ) when compared to vegetation surface temperature data. They used only four ground calibration targets, supporting our findings that even a small number of targets may be sufficient for producing robust temperature estimates. Likewise they attributed differences between $\mathrm{UAV}$ and validation surface temperature data to uncertainties in the validation data. Other studies used between 10 and 19 ground calibration points but provided no data to validate their calibration of non-radiometric UAV TIR cameras [34,35]. We noted that the accuracies achieved here are comparable to the manufacturer stated accuracies for the radiometric version of this camera (Vue Pro R $640, \pm 5^{\circ} \mathrm{C}$ ).

\subsection{Challenges and Applicability}

Although we found that a simple empirical line calibration could produce surface temperature estimates accurate to within a few degrees Celsius, this may not be sufficient for many applications within ecophysiology where much smaller temperature differences are expected. We have highlighted ways in which the accuracy of these measurements could be increased, through specific UAV sampling protocols (summarized in Figure 10), more extensive radiometric calibration procedures or post-processing operations. Nevertheless, we noted that some of these operations may involve significant investments of time, as well as demand resources that are not easily accessible (e.g., blackbody and climate chamber for rigorous radiometric calibration). Furthermore, there are some eccentricities in the performance of the UAV TIR camera we tested that we have not successfully explained or that may be difficult to rectify even with further work. For example, not having measurements of camera sensor temperature is a major limitation to more extensive calibration or understanding camera performance during flight. We therefore suggest that purchasing a radiometric UAV TIR camera with a relatively high $\left( \pm 2{ }^{\circ} \mathrm{C}\right)$ accuracy is worthwhile for applications where small differences in absolute temperatures are the focus of interest, despite the higher monetary cost involved. Within ecophysiology, such applications could include estimating evaporation [8], assessing tree health $[9,38]$, mapping vegetation water stress $[3,4,7]$, and improving vegetation carbon flux modelling [39]. 
There are five main sources of error when deriving temperature values from TIR cameras: radiometric calibration $[14,16]$, the influence of the sensor temperature (and temperature from other parts of the camera interior; $[13,15,17,22])$, vignetting [19], non-uniformity noise [40] and the correction for atmospheric effects, target emissivity and distance from the target [2,19-21]. In this study, we estimated error values of $\pm 5^{\circ} \mathrm{C}$ for the radiometric calibration, up to $\pm 20^{\circ} \mathrm{C}$ for the influence of sensor temperature (depending on whether the camera was under stable or changing ambient conditions), between $\pm 1.2-2.6{ }^{\circ} \mathrm{C}$ for vignetting (in field and experimental conditions) and $\leq-1.25{ }^{\circ} \mathrm{C}$ for the difference in emissivity between the TCPs and target vegetation. All five sources of error can be present in both non-radiometric and radiometric cameras. Given that all UAV TIR cameras use similar uncooled microbolometer systems, similar temperature-sensitivity related problems are expected for all other UAV TIR cameras. The severity of the error will depend on the camera model, the manufacturer (or user) calibration process and user input (e.g., input of target emissivity and atmospheric parameters as part of the radiometric model in radiometric TIR cameras). Therefore, the recommendations summarized in Figure 10 apply not only to non-radiometric but also to radiometric UAV TIR cameras, some of which also have relatively low accuracies.

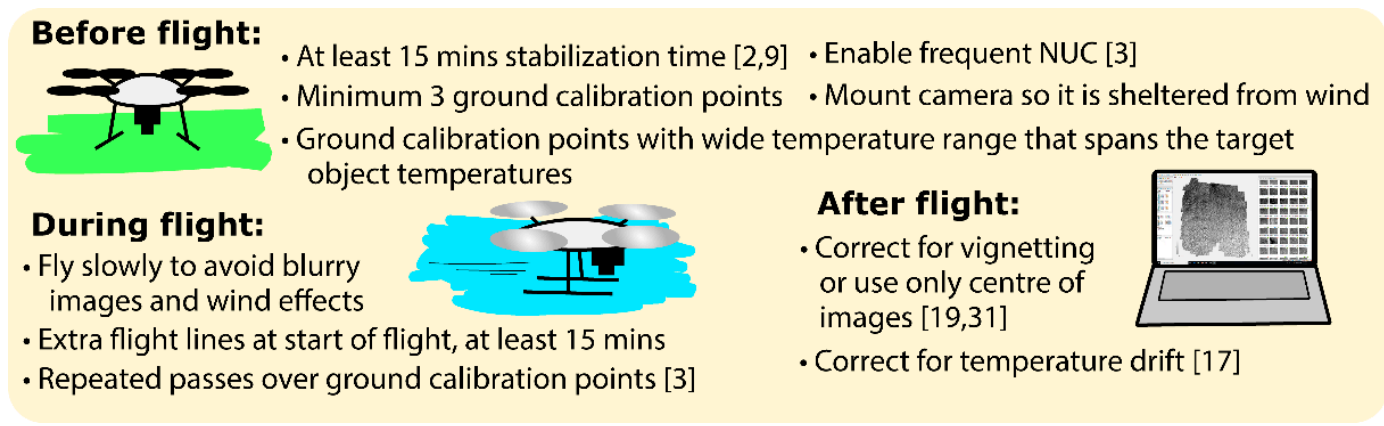

Figure 10. Recommendations for deriving robust data from an uncalibrated UAV TIR camera based on the results of this study and the literature.

\subsection{Future Work}

More research is needed to develop robust protocols for the calibration of UAV TIR cameras. Aasen et al. [31] recently published a comprehensive review of measurement procedures and data correction processes for UAV spectroscopy and many of these findings also provide a solid foundation for UAV TIR data collection. To date, there is still a lack of UAV sampling protocols specific to UAV TIR applications. These should account for the pronounced temperature-sensitivity of output data as well as for difficulties in producing orthomosaics from TIR data that often produce low contrast and poor quality imagery $[16,28]$. They should also investigate the choice of parameters when constructing orthomosaics, particularly the way in which resampling is performed, what blending option is used, and how that may influence the final values in the orthomosaic (e.g., as seen in Aasen and Bolten [41] for hyperspectral imagery). Furthermore, there is a lack of understanding of directional viewing effects on UAV TIR data, though related work has been carried out for other types of UAV cameras [42], for TIR data from satellites [43], and for ground-based cameras [19].

\section{Conclusions}

We assessed the effects of sensor temperature dependency on the performance of an uncooled, non-radiometric UAV TIR camera. Our laboratory and field experiments revealed substantial, non-linear changes in camera response to varying ambient conditions as typically experienced during UAV flight. By performing a simple empirical line calibration, we produced temperature estimates with a $\pm 5{ }^{\circ} \mathrm{C}$ uncertainty range during UAV flight conditions and to within $1{ }^{\circ} \mathrm{C}$ during stable laboratory conditions. We concluded that higher accuracies with this type of camera could not be expected during UAV flight conditions unless greater time is spent on camera stabilization in the field (following our 
recommended UAV flight protocols) and data on camera sensor temperature are available to develop a more robust radiometric calibration. Without this data, the temperature dependency of the camera sensor cannot be fully corrected. Even the use of relative temperature differences (the main application for this type of camera) may be challenging given the complex response of camera output to changes in ambient conditions and the prominent vignetting effect visible across the sensor. We presented a set of best practices for UAV TIR camera sampling. We also highlighted the fact that further work is needed to develop robust TIR UAV sampling protocols that account for the low signal-to-noise ratio of TIR imagery and the impacts of target emissivity, atmospheric attenuation of TIR radiation and directional viewing effects on temperature estimation.

Author Contributions: Conceptualization, J.K., N.K., L.E.; methodology, J.K., N.K.; formal analysis, J.K., P.-O.O., N.K., L.M.; resources, N.K., L.E., L.K.; data curation, B.L., P.W., P.-O.O.; writing-original draft preparation, J.K.; writing-review and editing, all authors; supervision, N.K., L.E.; project administration, N.K., L.E., L.K.; and funding acquisition, N.K., L.E., L.K., J.K.

Funding: This research was funded by Svenska Forskningsrådet Formas, grant number 2016-01223, the Royal Geographical Society (with IBG) Dudley Stamp Memorial Award, the Engineering and Physical Sciences Research Council (EPSRC, UK), and by the European Cooperation in Science and Technology (COST) Action ES1309, STSM reference number ES1309-36593.

Acknowledgments: This article is based upon work from COST Action ES1309 OPTIMISE, supported by COST (European Cooperation in Science and Technology; www.cost.eu). The authors thank the University of Gothenburg for the contribution of materials and access to the Skogaryd Research Station, where field measurement campaigns were conducted. J.K. is grateful for the financial support from Swansea University (UK) during the first years of her PhD. Special thanks are also given to Jonas Nilsson for his invaluable contributions to the field measurement campaign and to Meelis Mölder for his assistance in the laboratory at Lund University.

Conflicts of Interest: The authors declare no conflicts of interest. The funders had no role in the design of the study; in the collection, analyses, or interpretation of data; in the writing of the manuscript, or in the decision to publish the results.

\section{Appendix A}

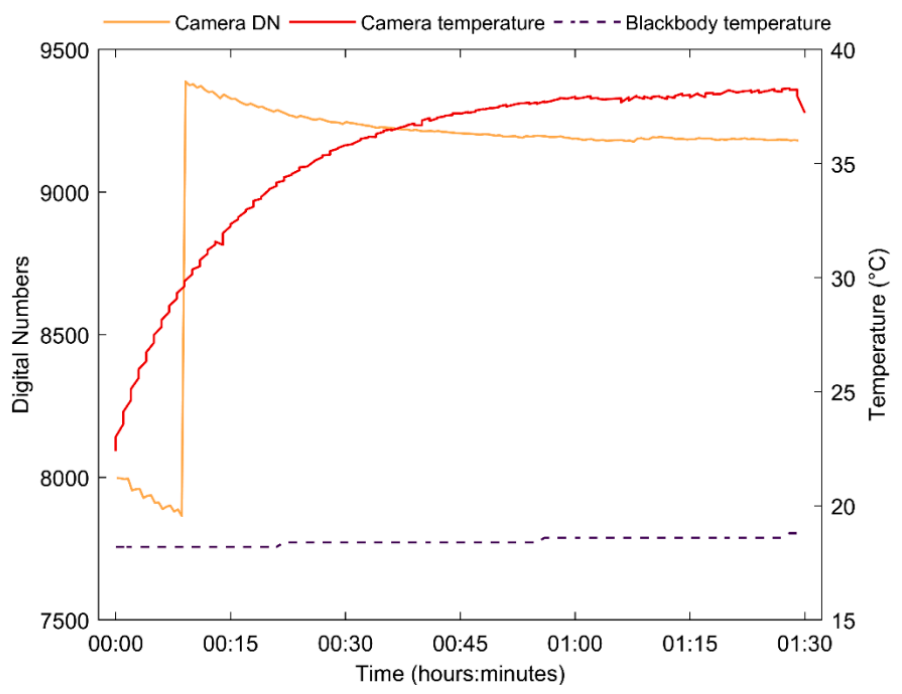

Figure A1. Repetition of the camera stabilization experiment: time series of mean camera digital number, temperature of the camera and blackbody temperature for the first $1.5 \mathrm{~h}$ after camera activation. 


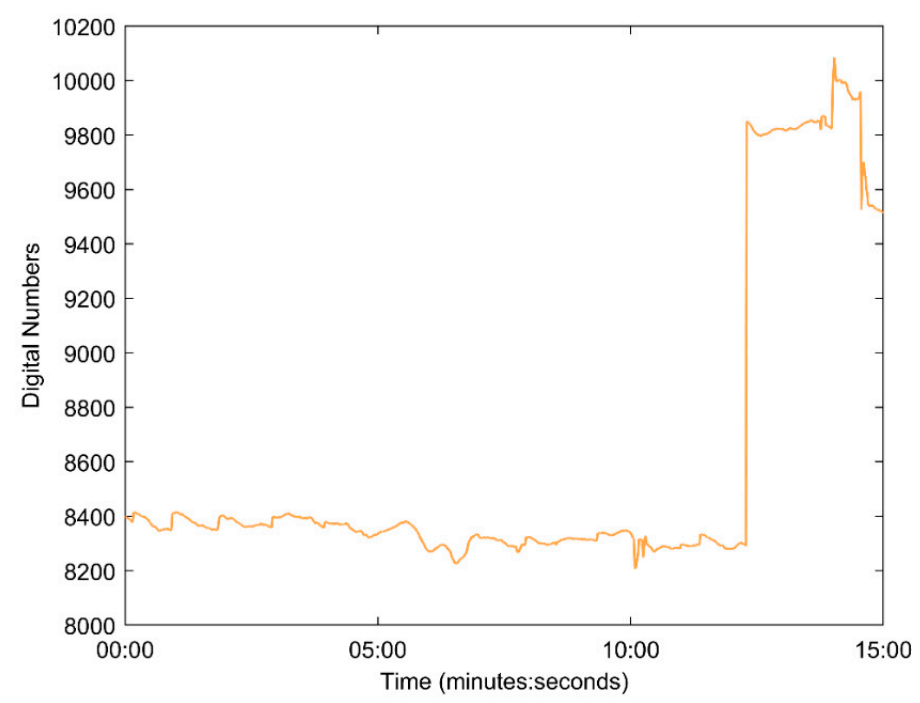

Figure A2. Time series of mean camera digital number from images recorded every second during the camera stabilization time before Flight A.

\section{References}

1. USGS. Landsat 8 Data Users Handbook; USGS: Sioux Falls, SD, USA, 2018.

2. Berni, J.A.J.; Zarco-Tejada, P.J.; Suarez, L.; Fereres, E. Thermal and Narrowband Multispectral Remote Sensing for Vegetation Monitoring From an Unmanned Aerial Vehicle. IEEE Trans. Geosci. Remote Sens. 2009, 47, 722-738. [CrossRef]

3. Gómez-Candón, D.; Virlet, N.; Labbé, S.; Jolivot, A.; Regnard, J.L. Field phenotyping of water stress at tree scale by UAV-sensed imagery: New insights for thermal acquisition and calibration. Precis. Agric. 2016, 17, 786-800. [CrossRef]

4. Gonzalez-Dugo, V.; Zarco-Tejada, P.; Berni, J.A.J.; Suárez, L.; Goldhamer, D.; Fereres, E. Almond tree canopy temperature reveals intra-crown variability that is water stress-dependent. Agric. For. Meteorol. 2012, 154-155, 156-165. [CrossRef]

5. Hoffmann, H.; Jensen, R.; Thomsen, A.; Nieto, H.; Rasmussen, J.; Friborg, T. Crop water stress maps for an entire growing season from visible and thermal UAV imagery. Biogeosciences 2016, 13, 6545-6563. [CrossRef]

6. Brenner, C.; Thiem, C.E.; Wizemann, H.D.; Bernhardt, M.; Schulz, K. Estimating spatially distributed turbulent heat fluxes from high-resolution thermal imagery acquired with a UAV system. Int. J. Remote Sens. 2017, 38, 3003-3026. [CrossRef] [PubMed]

7. Berni, J.A.J.; Zarco-Tejada, P.J.; Sepulcre-Cantó, G.; Fereres, E.; Villalobos, F. Mapping canopy conductance and CWSI in olive orchards using high resolution thermal remote sensing imagery. Remote Sens. Environ. 2009, 113, 2380-2388. [CrossRef]

8. Hoffmann, H.; Nieto, H.; Jensen, R.; Guzinski, R.; Zarco-Tejada, P.; Friborg, T. Estimating evaporation with thermal UAV data and two-source energy balance models. Hydrol. Earth Syst. Sci. 2016, 20, 697-713. [CrossRef]

9. Smigaj, M.; Gaulton, R.; Suarez, J.C.; Barr, S.L. Use of miniature thermal cameras for detection of physiological stress in conifers. Remote Sens. 2017, 9, 957. [CrossRef]

10. Sugiura, R.; Noguchi, N.; Ishii, K. Correction of low-altitude thermal images applied to estimating soil water status. Biosyst. Eng. 2007, 96, 301-313. [CrossRef]

11. Jensen, A.M.; Neilson, B.T.; McKee, M.; Chen, Y. Thermal remote sensing with an autonomous unmanned aerial remote sensing platform for surface stream temperatures. In Proceedings of the 2012 IEEE International Geoscience and Remote Sensing Symposium, Munich, Germany, 22-27 July 2012.

12. Stark, B.; Smith, B.; Chen, Y. Survey of thermal infrared remote sensing for Unmanned Aerial Systems. In Proceedings of the 2014 International Conference on Unmanned Aircraft Systems (ICUAS), Orlando, FL, USA, 27-30 May 2014.

13. Olbrycht, R.; Więcek, B.; De Mey, G. Thermal drift compensation method for microbolometer thermal cameras. Appl. Opt. 2012, 51, 1788-1794. [CrossRef] 
14. Budzier, H.; Gerlach, G. Calibration of uncooled thermal infrared cameras. J. Sensors Sens. Syst. 2015, 4, 187-197. [CrossRef]

15. Nugent, P.W.; Shaw, J.A.; Pust, N.J. Correcting for focal-plane-array temperature dependence in microbolometer infrared cameras lacking thermal stabilization. Opt. Eng. 2013, 52, 061304. [CrossRef]

16. Ribeiro-Gomes, K.; Hernández-López, D.; Ortega, J.F.; Ballesteros, R.; Poblete, T.; Moreno, M.A. Uncooled thermal camera calibration and optimization of the photogrammetry process for UAV applications in agriculture. Sensors 2017, 17, 2173. [CrossRef] [PubMed]

17. Mesas-Carrascosa, F.J.; Pérez-Porras, F.; de Larriva, J.E.M.; Frau, C.M.; Agüera-Vega, F.; Carvajal-Ramírez, F.; Martínez-Carricondo, P.; García-Ferrer, A. Drift correction of lightweight microbolometer thermal sensors on-board unmanned aerial vehicles. Remote Sens. 2018, 10, 615. [CrossRef]

18. Smigaj, M.; Gaulton, R.; Barr, S.L.; Suarez, J.C. Investigating the performance of a low-cost thermal imager for forestry applications. In Proceedings of the Image and Signal Processing for Remote Sensing XXII, Edinburgh, UK, 26-28 September 2016.

19. Meier, F.; Scherer, D.; Richters, J.; Christen, A. Atmospheric correction of thermal-infrared imagery of the 3-D urban environment acquired in oblique viewing geometry. Atmos. Meas. Tech. 2011, 4, 909-922. [CrossRef]

20. Aubrecht, D.M.; Helliker, B.R.; Goulden, M.L.; Roberts, D.A.; Still, C.J.; Richardson, A.D. Continuous, long-term, high-frequency thermal imaging of vegetation: Uncertainties and recommended best practices. Agric. For. Meteorol. 2016, 228, 315-326. [CrossRef]

21. Hammerle, A.; Meier, F.; Heinl, M.; Egger, A.; Leitinger, G. Implications of atmospheric conditions for analysis of surface temperature variability derived from landscape-scale thermography. Int. J. Biometeorol. 2017, 61, 575-588. [CrossRef]

22. Olbrycht, R.; Więcek, B. New approach to thermal drift correction in microbolometer thermal cameras. Quant. Infrared Thermogr. J. 2015, 12, 184-195. [CrossRef]

23. FLIR Tech Note: Radiometric Temperature Measurements. Available online: https://www.flir.com/ globalassets / guidebooks/suas-radiometric-tech-note-en.pdf (accessed on 20 November 2018).

24. Palmer, J.M. The measurement of transmission, absorption, emission, and reflection. In Handbook of Optics_Volume II: Devices, Measurements and Properties; Bass, M., Van Stryland, E.W., Williams, D.R., Wolfe, W.L., Eds.; McGraw-Hill, Inc.: New York, NY, USA, 1995; pp. 25.1-25.25, ISBN 0-07-047974-7.

25. Mölder, M.; Kellner, E. Excess resistance of bog surfaces in central Sweden. Agric. For. Meteorol. 2002, 112, 23-30. [CrossRef]

26. Kettridge, N.; Baird, A. Modelling soil temperatures in northern peatlands. Eur. J. Soil Sci. 2008, 59, 327-338. [CrossRef]

27. Royer, A.; Bussieres, N.; Goita, K. Characterization of land surface thermal structure from NOAA-AVHRR data over a northern ecosystem. Remote Sens. Environ. 1997, 60, 282-298.

28. Maes, W.H.; Huete, A.R.; Steppe, K. Optimizing the processing of UAV-based thermal imagery. Remote Sens. 2017, 9, 476. [CrossRef]

29. Mitchell, H.B. Image Fusion: Theories, Techniques and Applications; Springer-Verlag: Berlin/Heidelberg, Germany, 2010; Volume 134, ISBN 9783642112157.

30. Goldman, D.B. Vignette and Exposure Calibration and Compensation. IEEE Trans. Pattern Anal. Mach. Intell. 2010, 32, 2276-2288. [CrossRef] [PubMed]

31. Aasen, H.; Honkavaara, E.; Lucieer, A.; Zarco-Tejada, P.J. Quantitative remote sensing at ultra-high resolution with UAV spectroscopy: A review of sensor technology, measurement procedures, and data correction work flows. Remote Sens. 2018, 10, 1091. [CrossRef]

32. Pix4D SA. Pix4Dmapper 4.1 User Manual. Available online: https://support.pix4d.com/hc/en-us/articles/ 204272989-Offline-Getting-Started-and-Manual-pdf- (accessed on 20 November 2018).

33. Zhou, H.X.; Lai, R.; Liu, S.Q.; Jiang, G. New improved nonuniformity correction for infrared focal plane arrays. Opt. Commun. 2005, 245, 49-53. [CrossRef]

34. Jensen, A.M.; Mckee, M. Calibrating thermal imagery from an unmanned aerial vehicle AggieAir. In Proceedings of the 2013 IEEE International Geoscience and Remote Sensing Symposium, Melbourne, VIC, Australia, 21-26 July 2013.

35. Turner, D.; Lucieer, A.; Malenovský, Z.; King, D.H.; Robinson, S.A. Spatial co-registration of ultra-high resolution visible, multispectral and thermal images acquired with a micro-UAV over antarctic moss beds. Remote Sens. 2014, 6, 4003-4024. [CrossRef] 
36. Horny, N. FPA camera standardisation. Infrared Phys. Technol. 2003, 44, 109-119. [CrossRef]

37. Kaltenbach, H.-M. A Concise Guide to Statistics; Springer: Berlin/Heidelberg, Germany, 2012; ISBN 9783642235023.

38. Smigaj, M.; Gaulton, R.; Barr, S.L.; Suárez, J.C. UAV-Borne thermal imaging for forest health monitoring: Detection Of disease-induced canopy temperature increase. Int. Arch. Photogramm. Remote Sens. Spat. Inf. Sci. 2015, 40, 349-354. [CrossRef]

39. Kim, Y.; Still, C.J.; Hanson, C.V.; Kwon, H.; Greer, B.T.; Law, B.E. Canopy skin temperature variations in relation to climate, soil temperature, and carbon flux at a ponderosa pine forest in central Oregon. Agric. For. Meteorol. 2016, 226-227, 161-173. [CrossRef]

40. Goodall, T.R.; Bovik, A.C.; Paulter, N.G. Tasking on natural statistics of infrared images. IEEE Trans. Image Process. 2016, 25, 65-79. [CrossRef]

41. Aasen, H.; Bolten, A. Multi-temporal high-resolution imaging spectroscopy with hyperspectral 2D imagers-From theory to application. Remote Sens. Environ. 2018, 205, 374-389. [CrossRef]

42. Burkart, A.; Aasen, H.; Alonso, L.; Menz, G.; Bareth, G.; Rascher, U. Angular dependency of hyperspectral measurements over wheat characterized by a novel UAV based goniometer. Remote Sens. 2015, 7, 725-746. [CrossRef]

43. Duffour, C.; Lagouarde, J.P.; Roujean, J.L. A two parameter model to simulate thermal infrared directional effects for remote sensing applications. Remote Sens. Environ. 2016, 186, 250-261. [CrossRef]

(c) 2019 by the authors. Licensee MDPI, Basel, Switzerland. This article is an open access article distributed under the terms and conditions of the Creative Commons Attribution (CC BY) license (http://creativecommons.org/licenses/by/4.0/). 